\title{
EVALUACIÓN DEL ESTADO SUCESIONAL DE UN BOSQUE SUBTROPICAL DE QUEBRADAS EN EL NORTE DE URUGUAY
}

\author{
Iván A. Grela ${ }^{1}$
}

Recebido em 17/12/01. Aceito em 5/11/2002

\begin{abstract}
RESUMO - (Avaliação do estádio sucessional de uma floresta subtropical de "quebrada" na região norte do Uruguai). O objetivo do trabalho foi determinar o estado sucessional de uma floresta subtropical logo após esta ter sido explorada seletivamente com fins madeireiros. Comparou-se a estrutura atual da vegetação com a estrutura de uma zona testemunha contígua. A comparação foi feita com base nos seguintes parâmetros fitossociológicos: dominância, abundância (densidade), frequiência e índice do valor de importância. O número de espécies encontradas foi de 30 na zona em sucessão e 24 na zona testemunha, o índice de similaridade de Czekanowski entre as duas foi igual a 0,83. Das dez espécies mais importantes de cada zona, sete são comuns e possuem valor de importância similar. Da análise conclui-se que a comunidade estudada possui o potencial para se regenerar e retornar ao estado de evolução anterior à perturbação.
\end{abstract}

Palavras-chave - Uruguai, sucessão natural, fitossociologia, valor de importância, exploração seletiva de floresta

RESUMEN - (Evaluación del estado sucesional de un bosque subtropical de quebradas en el norte de Uruguay). Con el objetivo de determinar el estado sucesional de una formación forestal subtropical luego de haber sido explotada selectivamente con fines madereros, se comparó la estructura actual de la misma con la de una zona contigua que no fue explotada y que se tomó como testigo. Para la comparación se tuvieron en cuenta los siguientes parámetros fitosociológicos: dominancia, abundancia (densidad), frecuencia e índice de valor de importancia. El número especies detectadas fue treinta en la zona en sucesión y veinticuatro en la testigo, y el indice de similitud de Czekanowski entre ambas de 0.83 . De las diez especies más importantes en cada zona, siete son compartidas y poseen valores de importancia similares. De acuerdo a los diferentes análisis efectuados se concluye que la comunidad estudiada cuenta con el potencial para regenerase y retornar a su estado de evolución anterior.

Palabras clave - Uruguay, sucesión natural, fitosociología, valor de importancia, explotación selectiva de bosque

\section{Introducción}

En el Uruguay la vegetación arbórea ocupa alrededor del $3 \%$ de su superficie, con un total de 224 especies arbóreas y arborescentes (Lombardo 1964), aunque de acuerdo a estudios más recientes, esta cifra supera las 250 considerando también las especies leñosas arbustivas (Marchesi com. pers.).

\footnotetext{
1 Departamento de Producción Forestal y Tecnología de la Madera, Facultad de Agronomía, Universidad de la República, Av. Garzón 780, Montevideo, Uruguay, CP:12.900 (iagrela@adinet.com.uy)
} 
Las formaciones arbóreas se encuentran prácticamente en todo el territorio, principalmente en los márgenes de los numerosos cursos de agua (bosques ribereños o de galería), en las laderas rocosas de las zonas escarpadas (serranías), en las planicies adyacentes al río Uruguay ("algarrobales" y "blanqueales") y en las abruptas depresiones formadas en el límite oriental de las coladas basálticas, conocidas en la terminología vernácula como "quebradas" que tienen lugar en el norte y noreste del país.

En estas últimas existe un tipo muy particular de vegetación arbórea de características subtropicales, con una mayor diversidad de especies y formas de vida en comparación con otras formaciones arbóreas del país.

Estos bosques se constituyen en una interesante vía de entrada de elementos florísticos típicos de la provincia fitogeográfica Paranense (Cabrera e Willink 1973; Chebataroff 1960), hecho que también se observa en la vegetación del río Uruguay. Por otra parte son las zonas que han conservado mejor sus características originales debido a la mayor dificultad de acceso (Brussa et al. 1993).

En otras zonas del país, en particular al sur del río Negro, las vegetación leñosa ha sido explotada en forma muy intensa e indiscriminada en épocas de crisis energética. Sin embargo, excepto en las situaciones en que se introdujo definitivamente un nuevo uso de la tierra (como la ganadería y la agricultura), las mismas se han regenerado, recuperando el área que ocupaban previamente.

En Uruguay existen muy escasos (prácticamente nulos) antecedentes que permitan comparar las características actuales de las comunidades forestales con la situación original en cuanto a composición florística, importancia relativa de las diferentes especies, comportamiento de la regeneración natural, etc. Tampoco existen estudios relacionados con la dinámica de éstas comunidades tanto en su condición natural como bajo manejo silvícola. En algunos casos se ha realizado estudios de la fitosociolgía de comunidades forestales que servirán de base para futuras comparaciones (Berterreche et al. 1991; Bonifacino et al. 1998; Brussa et al. 1993; Firpo et al. 1997). Por otra parte, en Uruguay no se han realizado experiencias de restauración de bosques nativos ni investigaciones serias sobre el comportamiento de los mismos cuando se encuentran sometidos a perturbaciones (Brussa et al. 1993).

Con el objetivo de evaluar el estado de la sucesión de una selva de quebrada ubicada en el Departamento de Rivera luego de haber sido talada en forma selectiva, se evaluaron dos sectores contiguos de la misma, uno que fue sometido a la intervención mencionada, y otro que mantuvo las características originales que fue tomado como testigo.

\section{Material y metodos}

Area de estudio - abarca un sector de 5ha ubicado en las nacientes del río Lunarejo (Departamento de Rivera), el que forma parte del sistema denominado "frente de cuesta basáltica", es decir el límite oriental de las extensas coladas basálticas que cubren parte del Uruguay y el sur de Brasil, en la zona de contacto con las formaciones de areniscas triásicas y jurásicas (Fig. 1). Las coordenadas geográficas del punto central son $31^{\circ} 09^{\prime} \mathrm{S}$ y $55^{\circ} 48^{\prime} \mathrm{W}$. El terreno es escarpado con abruptas depresiones (quebradas) actualmente ocupadas por el tipo de vegetación mencionado.

Parte de esta zona fue explotada en una sola oportunidad con fines madereros 13 años antes de la realización del presente trabajo. Se realizó un tala selectiva de las especies más valiosas como Luehea divaricata, Ocotea acutifolia, y Nectandra megapotamica principalmente. Esta actividad provocó una perturbación importante debido a la alta remoción de biomasa, pero en área relativamente pequeña. 


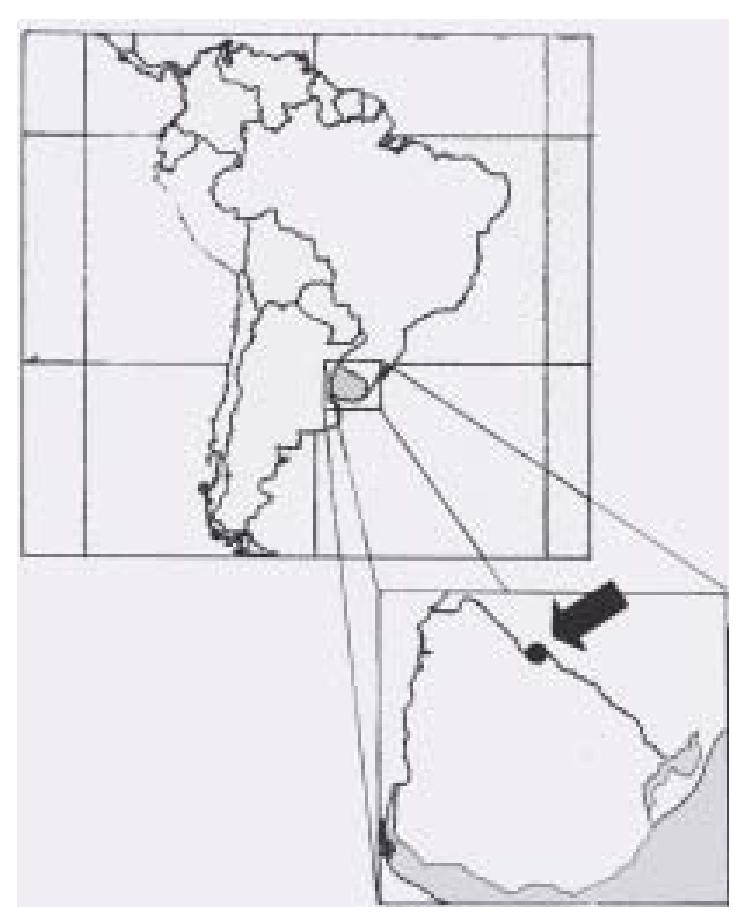

Figura 1. Ubicación del área de estudio.

La actividad maderera fue abandonada antes de completar el área prevista, quedando inalterada parte de la misma (la que se utilizó como testigo para comparar la evolución de la comunidad). Esto provocó el rápido inicio de una sucesión que llevó a la cobertura total del dosel arbóreo en poco tiempo. No obstante la regeneración de la cubierta arbórea, es necesario determinar si la misma posee características similares a la original o no.

Muestreo - el relevamiento de las especies arbóreas se realizó en cinco parcelas de $500 \mathrm{~m}^{2}$ $(20 \times 25 \mathrm{~m})$ en cada uno de los dos sitios. Estas parcelas se dividieron en subparcelas de $100 \mathrm{~m}^{2}$ a los efectos de la determinación de los valores de frecuencia. En estas parcelas se contaron todos los ejemplares con más de $3 \mathrm{~m}$ de altura y más de $3 \mathrm{~cm}$ de diámetro a la altura del pecho (DAP). Los individuos rebrotados a una altura menor a $1.3 \mathrm{~m}$ se contaron como uno a los efectos de la abundancia (densidad) y se midieron los diámetros de todos los rebrotes para el cálculo de la dominancia. La regeneración natural se estudió mediante 25 parcelas de $1 \mathrm{~m}^{2}$ en cada zona, considerándose como tal a todos los ejemplares que no cumplen las características mencionadas anteriormente.

El reconocimiento de las especies se efectuó en el área de estudio, exceptuando algunos casos dudosos en los que se recolectaron muestras para ser determinadas en el laboratorio. Las mismas se incorporaron a la colección del Herbario Bernardo Rosengurtt de la Facultad de Agronomía (MVFA).

Los parámetros utilizados para caracterizar los sitios fueron los siguientes (Curtis 1950; Curtis y McIntosh 1951; Finol 1971; Kent y Coker 1994; Matteuci y Colma 1982; MuellerDombois y Ellenberg 1974):

Dominancia (D): $\mathrm{D}_{\mathrm{i}}=\mathrm{Ab}_{\mathrm{i}} / \mathrm{S}\left(\mathrm{m}^{2} / \mathrm{ha}\right)$

$$
\mathrm{DR}_{\mathrm{i}}=\left(\mathrm{D}_{\mathrm{i}} / \Sigma \mathrm{D}_{\mathrm{i}}\right) * 100
$$$$
\mathrm{i}=1 . . \mathrm{n}
$$

$\mathrm{Ab}=$ sección del fuste a $1.3 \mathrm{~m}$ de altura $\left(\mathrm{m}^{2}\right)$

$\mathrm{DR}=$ dominancia (densidad) relativa de la especie i respecto de la dominancia total de la comunidad

$\mathrm{i}=$ especies de la comunidad, $1 \ldots \mathrm{n}$

$\mathrm{S}=$ superficie (ha)

Abundancia ${ }^{2}$

$$
\begin{aligned}
& (A): A_{i}=N_{i} / S \\
& A_{i}=\left(A_{i} / \sum A_{i}\right) * 100 \\
& i=1 . . n
\end{aligned}
$$

$\mathrm{AR}=$ abundancia relativa de la especie $\mathrm{i}$ respecto a la abundancia total

$\mathrm{Ni}=$ número de individuos de la especie $\mathrm{i}$

$\mathrm{S}=$ superficie (ha)

$\mathrm{i}=$ especies de la comunidad, $1 \ldots \mathrm{n}$

Frecuencia $(\mathrm{F}): \mathrm{F}_{\mathrm{i}}=\mathrm{P}_{\mathrm{i}} / \mathrm{NP}$

$$
\mathrm{FR}_{\mathrm{i}}=\underset{\mathrm{i}=1 \ldots \mathrm{n}}{\left(\mathrm{F}_{\mathrm{i}} / \Sigma \mathrm{F}_{\mathrm{i}}\right)} * 100
$$

$\mathrm{FR}=$ frecuencia relativa de la especie i respecto a la frecuencia total

$\mathrm{Pi}=\mathrm{N}$. de parcelas en que aparece la especie $\mathrm{i}$

$\mathrm{NP}=\mathrm{N}$. total de parcelas

$\mathrm{i}=$ especies de la comunidad, $1 \ldots \mathrm{n}$

\footnotetext{
2 En la literatura brasilera este parámetro se conoce como Densidad
} 
Indice de valor de importancia

$$
\begin{aligned}
& \text { (IVI): } \mathrm{IVI}_{\mathrm{i}}=\mathrm{A}_{\mathrm{i}} \%+\mathrm{D}_{\mathrm{i}} \%+\mathrm{F}_{\mathrm{i}} \% \\
& \mathrm{IVIR}_{\mathrm{i}}=\mathrm{IVI}_{\mathrm{i}} / 3 \\
& \mathrm{i}=\text { especies de la comunidad, } 1 \ldots \mathrm{n}
\end{aligned}
$$

Regeneración Natural (RN):

Para el cálculo de este parámetro se tomaron como plántulas aquellos ejemplares inferiores a $3 \mathrm{~m}$ de altura y/o con DAP menor a $3 \mathrm{~cm}$ Se calcularon los parámetros abundancia y frecuencia, el parámetro RN como la suma de ambos y $\mathrm{RNR}_{\mathrm{i}}$ como el porcentaje de $\mathrm{RN}$ de cada especie de la comunidad respecto del total.

Ambas zonas se compararon en base al índice de similitud cualitativo de Czekanowski (Wolda, 1981), conocido también como índice de Sørensen o de Dice definido como:

Indice de similitud $\left(\operatorname{IS}_{\mathrm{c}}\right)=2 \mathrm{c} /(\mathrm{a}+\mathrm{b})$

$\mathrm{a}=\mathrm{N}$. de especies de la zona 1

$\mathrm{b}=\mathrm{N}$. de especies de la zona 2

$\mathrm{c}=\mathrm{N}$. de especies en común

\section{Resultados y discusión}

El número de especies con individuos adultos fue de 29 y 30 en la zona en sucesión y testigo respectivamente (Tab. 1, 2 y 3) y el IS entre ambas fue de 0.84 , es decir que presentan una alta similitud florística

La composición florística y estructura fitosociológica de ambas comunidades son a su vez similares a las obtenidas por Brussa et al. (1993) y Bonifacino et al. (1998) en bosques pertenecientes al mismo sistema de quebradas. Los valores del $\mathrm{IS}_{\mathrm{c}}$ con respecto a esas comunidades es de 0.78 y 0.90 respectivamente cuando se considera el testigo, y de 0.79 y 0.73 respectivamente al considerar la zona en sucesión, teniendo en cuenta exclusivamente las especies con ejemplares adultos.

Las tablas 2 y 3 muestran grosso modo que la zona en sucesión presenta una estructura similar a la zona testigo, ya que comparten 3 de las 5 especies más importantes (es decir las que acumulan el $60 \%$ del IVI).
Tomando en cuenta las diez especies más importantes en cada caso (86.6 y $77.8 \%$ del IVI respectivamente) las especies en común son siete. A su vez los valores del IVIR de estas especies comunes son similares y siempre en el mismo orden de magnitud.

Es notorio sin embargo el aumento en la importancia de Celtis iguanaea, Cestrum parquii, y Zanthoxylum hiemale en las zonas en regeneración, pasando de valores de IVIR de $2.12,0.86$ y 0 a $12.48,4.89$ y 3.03 respectivamente. Celtis iguanaea es una especie apoyante $\mathrm{y}$ trepadora que habitualmente forma parte de estos bosques aunque raramente en forma tan importante como se observa en este caso, donde ha actuado como especie pionera. El caso contrario es el de Ocotea pulchella que decreció notoriamente, pasando de 7.21 a 1.6 del IVIR.

Debido a que el IVIR puede ocultar diferencias importantes entre las comunidades por el hecho de utilizar porcentajes, se analizaron algunos de los parámetros en forma absoluta. La tablas 4 muestra los valores de abundancia y dominancia respectivamente y los mismos reflejan una situación inversa para cada situación y cada parámetro.

Mientras que la dominancia total pasa de $50.06 \mathrm{~m}^{2} /$ ha en la zona testigo a $30.25 \mathrm{~m}^{2} /$ ha en la regeneración, la abundancia pasa de 2.036 individuos/ha a 4.364 individuos/ha es decir que en la zona en regeneración existen más individuos pero de mucho menor tamaño (más jóvenes). En este sentido hay comportamientos diferenciales según las especies. Ocotea acutifolia mostró la particularidad de un aumento ambos parámetros, explicado quizás por el éxito de las plántulas para establecerse luego de la perturbación y obtener rápidos incrementos diamétricos, aunque en general las especies que fueron utilizadas comercialmente para la producción de madera (Luehea divaricata, Nectandra megapotamica $\mathrm{y}$ Ocotea pulchella) tuvieron una fuerte disminución de la dominancia, aún cuando se dio un aumento en la abundancia. 
Tabla 1. Lista de especies arbóreas detectadas en el bosque de quebradas del río Lunarejo, Departamento de Rivera.

Familia/Especie

ANACARDIACEAE

Lithraea molleoides Engl.

ARECACEAE

Syagrus romanzoffiana (Cham.) Glassman

CELASTRACEAE

Schaefferia argentinensis Speg.

ERYTHROXYLACEAE

Erythroxylum myrsinites A. St-Hil.

EUPHORBIACEAE

Manihot grahamii Hook.

Sebastiania brasiliensis Spreng.

Sebastiania commersoniana (Baill.) L.B.Sm. \& Downs

FABACEAE

Calliandra tweediei Benth.

ICACINACEAE

Citronella paniculata (Mart.) Howard

LAURACEAE

Nectandra megapotamica Mez

Ocotea acutifolia $\mathrm{Mez}$

Ocotea puberula $\mathrm{Mez}$

Ocotea pulchella Mart.

MYRSINACEAE

Myrsine coriacea (Sw.) R.Br. ex Roem. \& Schult.

MYRTACEAE

Blepharocalyx salicifolius (H.B.K.) Berg

Eugenia uniflora $\mathrm{L}$.

Myrcianthes pungens (Berg) Legrand

Myrrhinium atropurpureum var. octandrum Benth.

PHYTOLACCACEAE

Phytolacca dioica L.

POLYGONACEAE

Ruprechtia laxiflora Meisn.

RHAMNACEAE

Scutia buxifolia Reissek

ROSACEAE

Quillaja brasiliensis Mart.

RUTACEAE

Zanthoxylum hiemale A. St-Hil.

SAPINDACEAE

Allophylus edulis (A. St-Hil.) Radlk.

Cupania vernalis Cambess.

SAPOTACEAE

Pouteria salicifolia (Spreng.) Radlk.

SOLANACEAE

Cestrum parquii L'Her.

Solanum sanctae-catharinae Dunal
Tabla 1. (continuación)

Familia/Especie

STYRACEAE

Styrax leprosus Hook. \& Arn.

TILIACEAE

Luehea divaricata Mart.

ULMACEAE

Celtis iguanaea (Jacq.) Sarg.

Celtis tala Gillies ex Planch.

VERBENACEAE

Citharexylum montevidense (Spreng.) Moldenke

Los valores obtenidos para las restantes especies siguen en líneas generales este mismo patrón, lo cual además está indicando que el restablecimiento de la cobertura forestal se da a través de nuevos individuos lo que seguramente permitirá la regeneración de un bosque predominantemente fustal (primario), y no como sucede en gran parte de los bosques del país, en los que la regeneración predominante es a través del rebrote de las cepas cortadas, originando formaciones predominantemente tallares (Carrere 1990a y 1990b).

Sin embargo el análisis de la distribución del número de individuos por clases diamétricas de seis de las especies más importantes comunes a ambas zonas muestra diferencias en la situación de cada una. Cupania vernalis y Nectandra megapotamica presentan una distribución similar entre ambas situaciones, con un mayor número de individuos en las clases menores aunque es notorio el aumento de éstos en la zona en regeneración (Fig. 2, A y D respectivamente).

Ocotea acutifolia (Fig. 2 C) tiene un comportamiento diferente, con muy pocos ejemplares en la zona testigo (generalmente sobremaduros y atacados por hongos de la podredumbre de la madera). La tala parecería haber revertido en parte esta situación con un aumento notorio de los ejemplares jóvenes. La perturbación parecería haber favorecido también a Allophyllus edulis y Luehea divaricata 
Tabla 2. Parámetros fitosociológicos del bosque de quebradas en sucesión del río Lunarejo, Rivera. AR= abundancia (densidad) relativa; $\mathrm{DR}=$ dominancia relativa; $\mathrm{FR}=$ Frecuencia relativa; $\mathrm{IVIR}=$ índice de valor de importancia relativo; $\mathrm{RNR}=$ regeneración natural relativa.

\begin{tabular}{|c|c|c|c|c|c|}
\hline Especie & $\mathrm{AR}$ & DR & FR & IVIR & RNR \\
\hline Cupania vernalis & 23,56 & 21,75 & 8,62 & 17,97 & 22,18 \\
\hline Celtis iguanea & 25,30 & 4,20 & 7,93 & 12,48 & 8,17 \\
\hline Nectandra megapotamica & 15,67 & 11,37 & 8,62 & 11,89 & 14,96 \\
\hline Myrcianthes pungens & 3,76 & 15,18 & 6,55 & 8,50 & 12,85 \\
\hline Ocotea acutifolia & 3,57 & 14,03 & 6,21 & 7,94 & 4,64 \\
\hline Cestrum parquii & 5,68 & 2,44 & 6,55 & 4,89 & 1,14 \\
\hline Luehea divaricata & 3,85 & 6,26 & 4,48 & 4,86 & 0,54 \\
\hline Allophyllus edulis & 1,37 & 4,60 & 3,10 & 3,03 & 7,69 \\
\hline Zanthoxylum hiemale & 2,38 & 2,23 & 4,48 & 3,03 & 1,69 \\
\hline Sebastiania commersoniana & 1,92 & 2,65 & 4,48 & 3,02 & 2,23 \\
\hline Eugenia uniflora & 1,47 & 1,14 & 4,48 & 2,36 & 3,12 \\
\hline Schaefferia argentinensis & 1,47 & 1,28 & 3,10 & 1,95 & 5,35 \\
\hline Citharexylum montevidense & 1,47 & 0,90 & 3,45 & 1,94 & 1,58 \\
\hline Blepharocalyx salicifolius & 1,19 & 0,60 & 3,45 & 1,75 & 2,27 \\
\hline Myrsine coriacea & 1,10 & 1,36 & 2,41 & 1,63 & 0,00 \\
\hline Ocotea pulchella & 1,10 & 1,00 & 2,76 & 1,62 & 3,18 \\
\hline Calliandra tweediei & 1,01 & 0,29 & 2,76 & 1,35 & 2,18 \\
\hline Phytolacca dioica & 0,09 & 3,52 & 0,34 & 1,32 & 0,00 \\
\hline Ruprechtia laxiflora & 0,09 & 0,02 & 3,79 & 1,30 & 0,00 \\
\hline Manhiot grahamii & 0,64 & 0,63 & 2,07 & 1,11 & 0,00 \\
\hline Styrax leprosus & 0,82 & 0,19 & 2,07 & 1,03 & 0,45 \\
\hline Solanum sanctae-catharinae & 0,46 & 0,28 & 1,72 & 0,82 & 0,64 \\
\hline Myrrhinium atropurpureum var. octandrum & 0,64 & 0,09 & 1,72 & 0,82 & 0,00 \\
\hline Pouteria salicifolia & 0,18 & 1,56 & 0,69 & 0,81 & 0,00 \\
\hline Citronella paniculata & 0,18 & 1,38 & 0,69 & 0,75 & 0,00 \\
\hline Sin determinar & 0,37 & 0,08 & 1,38 & 0,61 & 3,40 \\
\hline Celtis tala & 0,09 & 0,79 & 0,34 & 0,41 & 0,00 \\
\hline Scutia buxifolia & 0,27 & 0,12 & 0,69 & 0,36 & 0,45 \\
\hline Quillaja brasiliensis & 0,18 & 0,04 & 0,69 & 0,31 & 0,00 \\
\hline Lithrea molleoides & 0,09 & 0,03 & 0,34 & 0,15 & 0,45 \\
\hline Ocotea puberula & 0,00 & 0,00 & 0,00 & 0,00 & 0,84 \\
\hline
\end{tabular}

(Fig. 2, B y E respectivamente) en los que se observa un notorio aumento de las clases de menor diámetro cuando en la situación original la distribución era uniforme entre las clases. Myrcianthes pungens (Fig. 2 F) tiene un comportamiento muy parecido en cuanto a la distribución de las clases diamétricas, aunque contrariamente a las especies anteriores con menor número de individuos en la zona en regeneración.

En todos estos casos se ha observado un aumento de las clases de menor diámetro, lo que resulta auspicioso para el futuro de la comunidad ya que esto permitiría la reposición de los árboles que fueron talados o que actualmente están sobremaduros. Por otra parte se observan problemas en algunas especies en la zona sin tala, con muy pocos ejemplares jóvenes.

Finalmente en la distribución del parámetro RNR calculado para las plántulas no se detectan grandes diferencias entre las dos situaciones estudiadas. Esta distribución es semejante a la del IVIR correspondiente a cada caso, lo que refuerza lo afirmado en el párrafo anterior. 
Tabla 3. Parámetros fitosociológicos del bosque de quebradas del río Lunarejo, Rivera, zona testigo. AR= abundancia (densidad) relativa; $\mathrm{DR}=$ dominancia relativa; $F R=$ Frecuencia relativa; IVIR= índice de valor de importancia relativo; $\mathrm{RNR}=$ regeneración natural relativa.

\begin{tabular}{lrrrrr}
\hline Especie & AR & DR & FR & IVIR & RNR \\
\hline Myrcianthes pungens & 19,45 & 19,51 & 14,79 & 17,92 & 8,77 \\
Nectandra megapotamica & 24,56 & 16,01 & 12,43 & 17,66 & 16,15 \\
Cupania vernalis & 25,34 & 6,92 & 14,79 & 15,69 & 21,60 \\
Ocotea pulchella & 3,14 & 12,41 & 6,51 & 7,35 & 5,93 \\
Luehea divaricata & 1,77 & 13,55 & 3,55 & 6,29 & 0,37 \\
Schaefferia argentinesis & 7,47 & 2,42 & 8,88 & 6,25 & 3,18 \\
Sebastiania commersoniana & 3,14 & 3,04 & 8,28 & 4,82 & 3,06 \\
Ocotea acutifolia & 0,79 & 10,76 & 1,18 & 4,24 & 1,51 \\
Pouteria salicifolia & 1,18 & 5,64 & 2,96 & 3,26 & 0,68 \\
Allophyllus edulis & 2,16 & 2,88 & 4,14 & 3,06 & 8,27 \\
Eugenia uniflora & 2,36 & 0,49 & 5,33 & 2,73 & 3,08 \\
Celtis iguanea & 2,95 & 1,05 & 2,37 & 2,12 & 5,13 \\
Calliandra tweediei & 1,38 & 0,08 & 4,14 & 1,87 & 3,18 \\
Citharexylum montevidensis & 0,39 & 1,73 & 1,18 & 1,10 & 0,37 \\
Myrsine coriacea & 0,59 & 1,47 & 1,18 & 1,08 & 1,05 \\
Cestrum parquii & 0,79 & 0,61 & 1,18 & 0,86 & 0,37 \\
Blepharocalyx salicifolius & 0,39 & 0,91 & 1,18 & 0,83 & 3,56 \\
Scutia buxifolia & 0,59 & 0,05 & 1,78 & 0,80 & 1,05 \\
Citronella paniculata & 0,39 & 0,03 & 1,18 & 0,54 & 1,41 \\
Sin determinar & 0,20 & 0,36 & 0,59 & 0,38 & 0,34 \\
Sebastiania brasiliensis & 0,39 & 0,05 & 0,59 & 0,35 & 3,28 \\
Myrrinium atropurpureum var. octandrum & 0,20 & 0,01 & 0,59 & 0,27 & 0,88 \\
Ocotea puberula & 0,20 & 0,01 & 0,59 & 0,27 & 4,01 \\
Styrax leprosus & 0,20 & 0,01 & 0,59 & 0,26 & 0,00 \\
Syagrus romanzoffiana & 0,00 & 0,00 & 0,00 & 0,00 & 0,98 \\
Quillaja brasiliensis & 0,00 & 0,00 & 0,00 & 0,00 & 0,68 \\
Erythroxylum myrsinites & 0,00 & 0,00 & 0,00 & 0,00 & 0,37 \\
Zanthoxylum hiemale & 0,00 & 0,00 & 0,00 & 0,00 & 0,37 \\
Solanum sanctae-catharinae & 0,00 & 0,00 & 0,00 & 0,00 & 0,37 \\
\hline & & & & &
\end{tabular}

Dada la importante regeneración natural que se ha constatado en la zona perturbada surge la necesidad de efectuar nuevos estudio respecto del comportamiento de la misma en toda la comunidad y para cada especie en particular.

Conclusiones - la utilización del parámetro IVIR muestra que no existen grandes diferencias en cuanto a las estructuras de las dos comunidades generadas luego de la tala. Las especies de mayor peso ecológico son Cupania vernalis, Nectandra megapotamica, Celtis iguanaea, Myrcianthes pungens y Ocotea acutifolia en la zona en regeneración; y Nectandra megapotamica, Cupania vernalis,
Myrcianthes pungens, Ocotea pulchella, y Schaefferia argentinesis en la zona testigo.

La principal diferencia la presentan Celtis iguanaea, Cestrum parquii y Zanthoxylum hiemale, quienes aumentan en forma notoria su importancia en la zona en regeneración, las restantes especies nombradas en el párrafo anterior presentan pesos ecológicos similares en cada situación.

El análisis de los valores absolutos de los parámetros dominancia y abundancia sí permite diferenciar claramente las dos situaciones ya que mientras que la dominancia total es aproximadamente un $60 \%$ mayor en la situación 

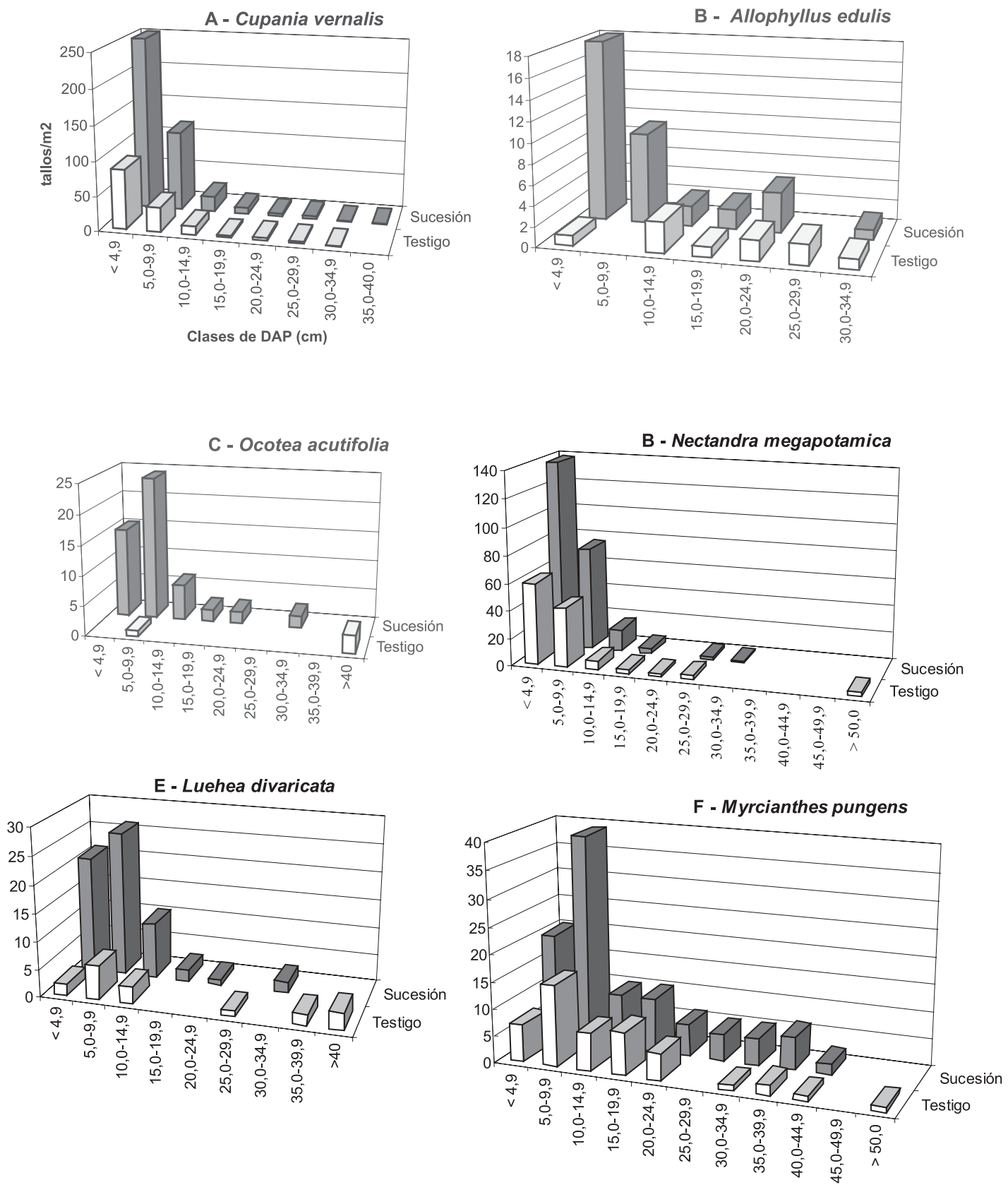

Figura 2. Distribución del número de tallos por clases diamétricas de las especies más importantes comunes a las zonas testigo y sucesión. 
Tabla 4. Valores absolutos de los parámetros abundancia $\left(\mathrm{A}_{\mathrm{abs}}\right)$ y dominancia $\left(\mathrm{D}_{\mathrm{abs}}\right)$ para las dos zonas estudiadas del bosque de quebradas del río Lunarejo, Rivera. $A_{a b s}=N$. individuos $/ \mathrm{ha} ; \mathrm{D}_{\mathrm{abs}}=\mathrm{m}^{2} / \mathrm{ha}$.

\begin{tabular}{|c|c|c|c|c|}
\hline \multirow[t]{2}{*}{ Especie } & \multicolumn{2}{|c|}{ Zona testigo } & \multicolumn{2}{|c|}{ Zona en sucesión } \\
\hline & $\mathrm{A}_{\mathrm{abs}}$ & $\mathrm{D}_{\mathrm{abs}}$ & $A_{a b s}$ & $\mathrm{D}_{\mathrm{abs}}$ \\
\hline Allophyllus edulis & 44 & 1,2132 & 60 & 1,39 \\
\hline Blepharocalyx salicifolius & 8 & 0,0076 & 52 & 0,1808 \\
\hline Calliandra tweediei & 28 & 0,0364 & 44 & 0,0864 \\
\hline Celtis iguanaea & 60 & 0,4536 & 1104 & 1,268 \\
\hline Celtis tala & - & - & 4 & 0,2376 \\
\hline Cestrum parquii & 16 & 0,3068 & 248 & 0,736 \\
\hline Citharexylum montevidense & 8 & 0,7384 & 64 & 0,2728 \\
\hline Citronella paniculata & 8 & 0,0156 & 8 & 0,418 \\
\hline Cupania vernalis & 516 & 5,3876 & 1028 & 6,5688 \\
\hline Eugenia uniflora & 48 & 0,2464 & 64 & 0,3432 \\
\hline Lithrea molleoides & - & - & 4 & 0,008 \\
\hline Luehea divaricata & 36 & 6,7852 & 168 & 1,8896 \\
\hline Manihot grahamii & - & - & 28 & 0,1896 \\
\hline Myrcianthes pungens & 396 & 2,4417 & 164 & 4,5848 \\
\hline Myrrhinium atropurpureum var. octandrum & 4 & 0,0052 & 28 & 0,0284 \\
\hline Myrsine coriacea & 12 & 0,5272 & 48 & 0,4116 \\
\hline Nectandra megapotamica & 500 & 8,0132 & 684 & 3,4776 \\
\hline Ocotea acutifolia & 16 & 1,4416 & 156 & 4,2384 \\
\hline Ocotea puberula & 4 & 0,0048 & - & - \\
\hline Ocotea pulchella & 64 & 6,2296 & 48 & 0,3024 \\
\hline Phytolacca dioica & - & - & 4 & 1,064 \\
\hline Pouteria salicifolia & 24 & 3,4656 & 8 & 0,47 \\
\hline Quillaja brasiliensis & - & - & 8 & 0,0132 \\
\hline Ruprechtia laxiflora & - & - & 4 & 0,0044 \\
\hline Schaefferia argentinensis & 152 & 0,8672 & 64 & 0,3864 \\
\hline Scutia buxifolia & 12 & 0,0228 & 12 & 0,0368 \\
\hline Sebastiania brasiliensis & 8 & 1,5208 & - & - \\
\hline Sebastiania commersoniana & 64 & 2,8216 & 84 & 0,8 \\
\hline Sin determinar & 4 & 0,178 & 16 & 0,0256 \\
\hline Solanum sanctae-catharinae & - & - & 20 & 0,0848 \\
\hline Styrax leprosus & - & - & 36 & 0,0584 \\
\hline Zanthoxylum hiemale & - & - & 104 & 0,6736 \\
\hline Total & 2036 & 50,058 & 4364 & 30,2492 \\
\hline
\end{tabular}

original, la zona en regeneración presenta más del doble de individuos/ha. Esto equivale a decir que la zona en sucesión presenta más ejemplares de menor tamaño (más jóvenes), y que la disminución de la dominancia provocada por la tala no ha sido compensada por el gran incremento en la abundancia.

Cupania vernalis, Nectandra megapotamica y Myrcianthes pungens mostraron una distribución diamétrica similar en las dos situaciones, con un mayor número de ejemplares jóvenes. Mientras las dos primeras aumentaron el número de individuos en la zona en regeneración, en la última disminuyó.

Ocotea acutifolia, Allophyllus edulis y Luehea divaricata no presentan distribuciones diamétricas adecuadas en la situación original, aunque en la zona en regeneración existe un aumento en las clases menores, en lo que parece en efecto positivo de la tala.

La regeneración natural, estudiada a través de la presencia de plántulas, presenta una 
estructura muy similar en ambas situaciones.

En función de todo lo anterior, puede inferirse que bajo las circunstancias descriptas (tipo de vegetación, ambiente, y tipo de perturbación) la comunidad estudiada presenta la capacidad de regenerarse en un estado similar al original. Evidentemente por tratarse de especies arbóreas el lapso transcurrido no permite sacar conclusiones definitivas, siendo necesario el seguimiento de las mismas. En particular surge la necesidad de establecer estudios más profundos sobre el comportamiento de la regeneración natural de las especies dominantes de la comunidad.

\section{Agradecimientos}

$\mathrm{El}$ autor agradece muy especialmente a los Ings. Agrs. Carlos Brussa y Fernanda Romero por su dirección, orientación y participación en las actividades de campo; a la Ing. Agr. (M.Sc.) Ana González por su colaboración en la traducción; al Dr. Jorge Waechter y a dos revisores anónimos por su lectura crítica, sugerencias y correcciones efectuadas.

\section{Referencias bibliográficas}

Berterreche, A.; De los Campos, D.; García, R. 1991. Estudio fitosociológico del Parque Nacional San Miguel, Departamento de Rocha. Tesis Ing. Agr. Universidad de la República - Facultad de Agronomía, Montevideo, Uruguay.

Bonifacino, M.; Cattaneo, M.; Profumo, L. 1998. Caracterización fitosociológica de un bosque de quebrada sobre el arroyo del Potrero. Cuchilla Negra, Departamento de Rivera. Tesis Ing. Agr. Facultad de Agronomía, Montevideo, Uruguay.

Brussa, C.; Majó, B.; Sans, C.; Sorrentino, A. 1993. Estudio fitosociológico del monte nativo en las nacientes del arroyo Lunarejo, departamento de Rivera. Boletín de Investigación 38. $32 \mathrm{p}$.
Cabrera, A. L. \& Willink, A. 1973. Biogeografía de América Latina. Secretaría General de la Organización de Estados Americanos. Programa Regional de Desarrollo Científico y Tecnológico, Departamento de Asuntos Científicos. Serie Biología 13, Washington D.C.

Carrere, R. 1990a. El bosque natural uruguayo: inventario y evolución del recurso. CIEDUR, Serie Investigaciones N. 78.

Carrere, R. 1990b. El bosque natural uruguayo: utilización tradicional y usos alternativos. CIEDUR, Serie Investigaciones N. 79.

Chebataroff, J. 1960. Tierra Uruguaya. Talleres Don Bosco, Montevideo.

Curtis, J. T. \& Mcintosh, R. P. 1950. The inter relation of certain analytic and synthetic phytosociological characters. Ecology 31: 434-455

Curtis, J. T. 1951. An upland forest continuum in the praire-forest border region of Wisconsin. Ecology 32: 476-496

Finol, H. 1971. Nuevos parámetros a considerarse en el análisis estructural de las selvas vírgenes tropicales. Revista Forestal Venezolana 13(21): $29-42$

Firpo, G.; Muniz, W.; Pepe, N.; Piriz, A. 1997. Estudio fitosociológico del monte nativo "Gruta de los Helechos". Departamento de Tacuarembó. Tesis Ing. Agr. Facultad de Agronomía, Montevideo, Uruguay.

Kent, M. \& Coker, P. 1994. Vegetation description and analysis. John Wiley and Sons Inc., Chichester.

Lombardo, A. 1964. Flora arbórea y arborescente del Uruguay. $2^{\text {a }}$. Concejo Municipal, Montevideo.

Matteuci, S. D. \& Colma, A. 1982. Metodología para el estudio de la vegetación. Secretaría General de la Organización de Estados Americanos. Serie de Biología 22, Washington, D.C.

Mueller-Dombois, D. \& Ellenberg, H. 1974. Aims and methods of vegetation ecology. John Wiley and Sons Ltd., New York.

Wolda, H. 1981. Similarity indices, sample size and diversity. Oecology 50: 296-302. 